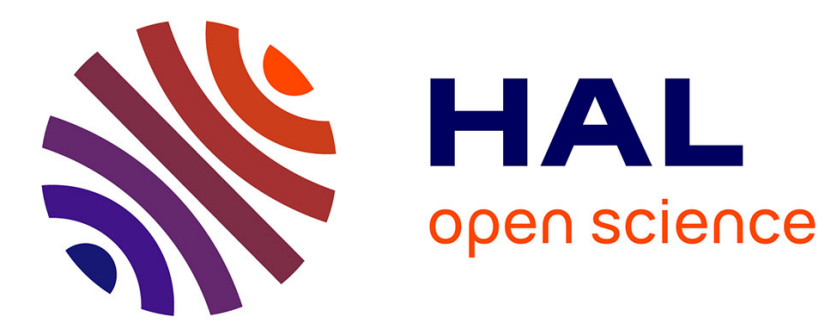

\title{
Theory of propagation of elastic waves in a fluid-saturated porous solid. I. Low-frequency range
}

Maurice A. Biot

\section{To cite this version:}

Maurice A. Biot. Theory of propagation of elastic waves in a fluid-saturated porous solid. I. Low-frequency range. Journal of the Acoustical Society of America, 1956, 28 (2), pp.168-178. 10.1121/1.1908239 . hal-01368666

\section{HAL Id: hal-01368666 https://hal.science/hal-01368666}

Submitted on 21 Sep 2016

HAL is a multi-disciplinary open access archive for the deposit and dissemination of scientific research documents, whether they are published or not. The documents may come from teaching and research institutions in France or abroad, or from public or private research centers.
L'archive ouverte pluridisciplinaire HAL, est destinée au dépôt et à la diffusion de documents scientifiques de niveau recherche, publiés ou non, émanant des établissements d'enseignement et de recherche français ou étrangers, des laboratoires publics ou privés. 


\title{
Theory of Propagation of Elastic Waves in a Fluid-Saturated Porous Solid. I. Low-Frequency Range
}

\author{
M. A. Biot* \\ Shell Development Company, RCA Building, New York, New York
}

(Received September 1, 1955)

\begin{abstract}
A theory is developed for the propagation of stress waves in a porous elastic solid containing a compressible viscous fluid. The emphasis of the present treatment is on materials where fluid and solid are of comparable densities as for instance in the case of water-saturated rock. The paper denoted here as Part $I$ is restricted to the lower frequency range where the assumption of Poiseuille flow is valid. The extension to the higher frequencies will be treated in Part II. It is found that the material may be described by four nondimensional parameters and a characteristic frequency. There are two dilatational waves and one rotational wave. The physical interpretation of the result is clarified by treating first the case where the fluid is frictionless. The case of a material containing a viscous fluid is then developed and discussed numerically. Phase velocity dispersion curves and attenuation coefficients for the three types of waves are plotted as a function of the frequency for various combinations of the characteristic parameters.
\end{abstract}

\section{INTRODUCTION}

$\mathbf{O}$ UR purpose is to establish a theory of propagation of elastic waves in a system composed of a porous elastic solid saturated by a viscous fluid. It is assumed that the fluid is compressible and may flow relative to the solid causing friction to arise. Part I which is presented here assumes that the relative motion of the fluid in the pores is of the Poiseuille type. As already pointed out by Kirchhoff this is valid only below a certain frequency which we denote by $f_{t}$, and which depends on the kinematic viscosity of the fluid and the size of the pores. Extension of the theory in the frequency range above $f_{t}$ will be presented in Part II. We have in mind particularly the application to cases where the fluid is a liquid, and we have therefore disregarded the thermoelastic effect. We include only materials such that the walls of the main pores are impervious and for which the pore size is concentrated around its average value. Extension to more general materials will be considered along with the thermoelastic effect in a later and more complete theory.

Development of the theory proceeds as follows. Section 2 introduces the concepts of stress and strain in the aggregate including the fluid pressure and dilatation. Relations are established between these quantities for static deformation in analogy with a procedure followed in the theory of elasticity for porous materials developed in reference 1 . Section 3 considers the dynamics of the material when the fluid is assumed to be without viscosity. This case is of practical interest since this represents the limiting behavior of wave propogation at very high frequency. It introduces the concepts of apparent masses and dynamic coupling between fluid and solid. The wave propogation in the absence of friction is analyzed in Secs. 4 and 5. It is found that there is one rotational wave and two dilatational waves. A remarkable property is the possible existence of a wave such that no relative motion occurs between fluid and solid.

\footnotetext{
* Consultant.

1 M. A. Biot, J. Appl. Phys. 26, 182 (1955).
}

This is obtained if a "dynamic compatibility" relation is verified between the elastic and dynamic constants. This is a case where dissipation due to fluid friction will disappear. In Sec. 6 the dynamic relations are derived with the addition of fluid viscosity and Sec. 7 derives the properties of the propagation of the waves when dissipation is present. It is found that the phase velocity of rotational waves increases slightly with the frequency $f$ while the absorption coefficient is proportional to the square of the frequency. All plots are presented nondimensionally by referring to a characteristic frequency $f_{c}$ which depends on the kinematic viscosity of the fluid and the pore diameter. The characteristic frequency $f_{c}$ may be considered as a frequency scale of the material, the nondimensional abscissa of the plots being the ratio $f / f_{c}$. There are two dilatational waves denoted as waves of the first and second kind. The waves of the second kind are highly attenuated. They are in the nature of a diffusion process, and the propagation is closely analogous to heat conduction. The waves of the first kind are true waves. The dispersion is practically negligible with a phase velocity increasing or decreasing with frequency depending on the mechanical parameters. The absorption coefficient is proportional to the square of the frequency as for the rotational waves. In cases close to the dynamic compatibility condition, the dispersion and attenuation of the waves of the first kind tend to vanish. The attenuation of this wave may therefore vary widely for materials of similar composition and may be larger or smaller than the attenuation of the rotational waves.

A beginning along the present lines was made by Frenkel. ${ }^{2} \mathrm{He}$ discusses the rotational and dilatational waves, but the subject is summarily treated and important features are neglected.

Sound absorption in material containing air was the object of extensive work by Zwikker and Kosten. ${ }^{3}$ Rotational waves are not considered, and simplified equations are used for the dilatational waves. The

\footnotetext{
${ }^{2}$ J. Frenkel, J. Phys. (U. S. S. R) 8, 230 (1944).

C. Zwikker and C. W. Kosten, Sound Absorbing Materials
} (Elsevier Publishing Company, Inc., New York, 1949). 
applications are presented mainly through the concept of impedance. The same approach is also emphasized by Beranek. ${ }^{4}$ The problem was also discussed by Morse under the simplifying assumption of a rigid solid. ${ }^{5}$ In the present series of papers we shall attempt to establish a more fundamental approach aimed at including successively all pertinent physical mechanisms in a theoretical and quantitative way as far as it seems possible.

\section{STRESS-STRAIN RELATIONS IN A POROUS ELASTIC SOLID CONTAINING A FLUID}

Consider a volume of the solid-fluid system represented by a cube of unit size. The stress tensor is separated into two parts.

(1) The force component acting on the solid parts of each face of the cube is one part. This is denoted as the tensor

$$
\left\{\begin{array}{lll}
\sigma_{x} & \tau_{z} & \tau_{y} \\
\tau_{z} & \sigma_{y} & \tau_{x} \\
\tau_{y} & \tau_{x} & \sigma_{z}
\end{array}\right\} .
$$

(2) The forces acting on the fluid part of each face of the cube are represented by

$$
\left\{\begin{array}{lll}
s & 0 & 0 \\
0 & s & 0 \\
0 & 0 & s
\end{array}\right\} .
$$

The scalar $s$ is proportional to the fluid pressure $p$ according to

$$
-s=\beta p,
$$

where $\beta$ is the fraction of fluid area per unit cross section. Note that $s$ is taken negative when the force acting on the fluid is a pressure while $\sigma_{x} \sigma_{y} \sigma_{z}$ are positive when the force in the solid is a tension.

It is important to call attention to the significance of this factor $\beta$. In the present analysis we assume that we are dealing with a statistically isotropic porous material in such a way that for all cross sections we always observe the same ratio of the fluid area to solid area. Hence the volume of fluid in a thin slab of thickness $d x$ is always a fraction $\beta$ of the total volume. This means that $\beta$ is identical with the quantity usually designated as porosity and denoted in the literature by the symbol $f$. (This symbol is used here to denote frequency.)

It should be mentioned that the porosity considered here is that which is connected with bulk motion of the fluid relative to the solid, i.e., that represented by the interconnecting void space. This is sometimes designated as "effective porosity." Sealed pore space is considered as part of the solid for our present purpose.

We denote the strain tensor in the solid by

$$
\left\{\begin{array}{ccc}
e_{x} & \frac{1}{2} \gamma_{z} & \frac{1}{2} \gamma_{y} \\
\frac{1}{2} \gamma_{z} & e_{y} & \frac{1}{2} \gamma_{x} \\
\frac{1}{2} \gamma_{y} & \frac{1}{2} \gamma_{x} & e_{z}
\end{array}\right\}
$$

' L. L. Beranek, J. Acoust. Soc. Am. 19, 556 (1947).

b R. W. Morse, J. Acoust. Soc. Am. 24, 696 (1952). with

$$
\begin{aligned}
e_{x} & =\partial u_{x} / \partial x, \text { etc. } \\
\gamma_{z} & =\frac{\partial u_{x}}{\partial y}+\frac{\partial u_{y}}{\partial x}, \text { etc. }
\end{aligned}
$$

where $u_{x}, u_{y}$, and $u_{z}$ are the components of the displacement vector of the solid. We must keep in mind that the material is of porous or granular structure and that we assume the size of the unit elements to be large compared to the pores. The displacement vector is defined here as the displacement of the material considered to be uniform and averaged over the element.

In a similar way we may talk of the average fluid displacement vector $U_{x} U_{y} U_{z}$ defined so that the product of this displacement by the cross-sectional fluid area represents the volume flow. The strain in the fluid is defined by the dilatation

$$
\epsilon=\frac{\partial U_{x}}{\partial x}+\frac{\partial U_{y}}{\partial y}+\frac{\partial U_{z}}{\partial z} .
$$

We now proceed to establish the relation between the stress and strain components of the solid-fluid aggregate as defined in the foregoing. For the time being we shall disregard all dissipative forces and assume that we are dealing with a conservative physical system which is in equilibrium when at rest. Any deformation is therefore a departure from a state of minimum potential energy. In the first approximation, therefore, this potential energy will be expressed by a positive definite quadratic form. The seven stress components $\sigma_{x} \sigma_{y} \sigma_{z} \tau_{x} \tau_{y} \tau_{z} s$ will then be linear functions of the seven strain components

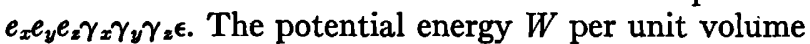
of aggregate is given by

$$
2 W=\sigma_{z} e_{x}+\sigma_{y} e_{y}+\sigma_{z} e_{z}+\tau_{x} \gamma_{x}+\tau_{y} \gamma_{y}+\tau_{z} \gamma_{z} .
$$

This quadratic form expressed in terms of the seven strain components involves in the more general case twenty-eight independent coefficients. The stress-strain relations may be expressed as

$$
\begin{gathered}
\sigma_{x}=\frac{\partial W}{\partial e_{x}} \quad \sigma_{y}=\frac{\partial W}{\partial e_{y}} \quad \sigma_{z}=\frac{\partial W}{\partial e_{z}} \\
\tau_{x}=\frac{\partial W}{\partial \gamma_{x}} \quad \tau_{y}=\frac{\partial W}{\partial \gamma_{y}} \quad \tau_{z}=\frac{\partial W}{\partial \gamma_{z}} . \\
s=\frac{\partial W}{\partial \epsilon}
\end{gathered}
$$

The seven-by-seven matrix of coefficient in the foregoing linear relations constitutes a symmetric matrix with twenty-eight distinct coefficients. ${ }^{3}$

These relations are very much simplified if we introduce the assumption that the solid-fluid system is 
statistically isotropic. In this case the principal stress and principal strain directions coincide. Referring the stresses and strains to these directions and taking into account the properties of symmetry of the material the stress-strain relations reduce to

$$
\begin{aligned}
\sigma_{I} & =B e_{I}+C\left(e_{I I}+e_{I I I}\right)+Q \epsilon \\
o_{I I} & =B e_{I I}+C\left(e_{I I I}+e_{I}\right)+Q \epsilon \\
\sigma_{I I I} & =B e_{I I I}+C\left(e_{I}+e_{I I}\right)+Q \epsilon \\
s & =Q^{\prime} e+R \epsilon
\end{aligned}
$$

with

$$
e_{I}+e_{I I}+e_{I I I}=e .
$$

Because of the existence of a potential energy the matrix of coefficients must be symmetric. This matrix is

$$
\left[\begin{array}{llll}
B & C & C & Q \\
C & B & C & Q \\
C & C & B & Q \\
Q^{\prime} & Q^{\prime} & Q^{\prime} & R
\end{array}\right] .
$$

Hence, we must have

$$
Q^{\prime}=Q
$$

In the isotropic case there are therefore four distinct elastic constants.

It is possible to write the stress-strain relations somewhat differently by introducing new constants $N$ and $A$ instead of $B$ and $C$. We write

$$
\begin{aligned}
\sigma_{I} & =2 N e_{I}+A e+Q \epsilon \\
\sigma_{I I} & =2 N e_{I I}+A e+Q \epsilon \\
\sigma_{I I I} & =2 N e_{I I I}+A e+Q \epsilon \\
s & =Q \epsilon+R \epsilon .
\end{aligned}
$$

In this form it is easy to express them for arbitrary directions of the coordinate axes by using the invariance of tensor relations. We find

$$
\begin{aligned}
\sigma_{x} & =2 N e_{x}+A e+Q \epsilon \\
\sigma_{y} & =2 N e_{y}+A e+Q \epsilon \\
\sigma_{z} & =2 N e_{z}+A e+Q \epsilon \\
\tau_{x} & =N \gamma_{x} \\
\tau_{y} & =N \gamma_{y} \\
\tau_{z} & =N \gamma_{z} \\
s & =Q e+R \epsilon
\end{aligned}
$$

with

$$
e=e_{x}+e_{y}+e_{z} .
$$

In examining the significance of these constants we notice that $A$ and $N$ correspond to the familiar Lamé coefficients in the theory of elasticity and are of positive sign.

The coefficient $N$ represents the shear modulus of the material. The coefficient $R$ is a measure of the pressure required on the fluid to force a certain volume of the fluid into the aggregate while the total volume remains constant. It must be of positive sign. The coefficient $Q$ is of the nature of a coupling between the volume change of the solid and that of the fluid. Its significance is illustrated by putting the fluid pressure equal to zero, in which case

$$
\epsilon=-Q e / R \text {. }
$$

Since a pressure on the solid must tend to produce a decrease in the porosity of the solid, $e$ and $\epsilon$ must be of opposite sign and $Q$ is positive.

One can devise experiments by which the four elastic coefficients may be measured statically. The shear modulus may be measured directly. The other coefficients are found by testing for stress versus strain confining the material by an impervious boundary in one test and a pervious boundary in the other. In the former test the fluid pressure must also be measured. The question remains, of course, to determine whether these static coefficients should be subject to a correction for application to dynamic phenomena. We shall investigate this question more thoroughly in a later paper.

\section{DYNAMIC RELATIONS IN THE ABSENCE OF DISSIPATION}

We now call our attention to the relation between stresses and acceleration in the absence of dissipative forces. It is convenient here to introduce the Lagrangian viewpoint and the concept of generalized coordinates. We again consider a unit cube of the aggregate as an element. The element is assumed to be small relative to the wavelength of the elastic waves and in turn the size of the pores is assumed small compared to the size of the element. The limitation of frequencies which is hereby introduced will turn out to be academic for most practical problems.

One important consequence of this assumption on the relative size of wavelength and pores is the fact that the microscopic velocity pattern is the same as if the fluid were incompressible. This follows from a general principle that a velocity field in compressible fluids approximates that of an incompressible fluid for obstacles which are small compared to the wavelength. Hence, the microscopic flow pattern of the fluid relative to the solid depends only on the direction of the relative flow and not on its magnitude. This assumption is valid if we neglect the viscosity and consider that we are dealing with a perfect fluid. In this case, the microscopic velocity field will be a linear function of the six average velocity components of the solid and the fluid. The Lagrangian coordinates are chosen as the six average displacement components of the solid and the fluid, i.e., respectively,

$$
u_{x}, u_{y}, u_{z}, U_{z}, U_{y}, U_{z} .
$$

The kinetic energy $T$ of the system per unit volume may 
be expressed as

$$
\begin{aligned}
2 T=\rho_{11} & {\left[\left(\frac{\partial u_{x}}{\partial t}\right)^{2}+\left(\frac{\partial u_{y}}{\partial t}\right)^{2}+\left(\frac{\partial u_{z}}{\partial t}\right)^{2}\right] } \\
& +2 \rho_{12}\left[\frac{\partial u_{x}}{\partial t} \frac{\partial U_{x}}{\partial t}+\frac{\partial u_{y}}{\partial t} \frac{\partial U_{y}}{\partial t}+\frac{\partial u_{z}}{\partial t} \frac{\partial U_{z}}{\partial t}\right] \\
& +\rho_{22}\left[\left(\frac{\partial U_{x}}{\partial t}\right)^{2}+\left(\frac{\partial U_{y}}{\partial t}\right)^{2}+\left(\frac{\partial U_{z}}{\partial t}\right)^{2}\right]
\end{aligned}
$$

This expression is based on the assumption that the material is statistically isotropic hence the directions $x, y, z$ are equivalent and uncoupled dynamically. Let us discuss the significance of the expression for the kinetic energy. The coefficients $\rho_{11} \rho_{22} \rho_{12}$ are mass coefficients which take into account the fact that the relative fluid flow through the pores is not uniform.

In discussing the significance of expression (3.2) we may, without loss of generality, consider a motion restricted to the $x$-direction. If we denote by $q_{x}$ the total force acting on the solid per unit volume in the $x$-direction and by $Q_{x}$, the total force on the fluid per unit volume, we derive from Lagrange's equations

$$
\begin{gathered}
\frac{\partial}{\partial t}\left(\frac{\partial T}{\partial \dot{u}_{x}}\right)=\frac{\partial^{2}}{\partial t^{2}}\left(\rho_{11} u_{x}+\rho_{12} U_{x}\right)=q_{x} \\
\frac{\partial}{\partial t}\left(\frac{\partial T}{\partial \dot{U}_{x}}\right)=\frac{\partial^{2}}{\partial t^{2}}\left(\rho_{12} u_{x}+\rho_{22} U_{x}\right)=Q_{x} .
\end{gathered}
$$

Before applying these relations (3.3) let us further discuss the nature of the coefficients $\rho_{11} \rho_{22} \rho_{12}$. Let us assume that there is no relative motion between fluid and solid. In this case

and

$$
u_{x}=U_{x}
$$

$$
2 T=\left(\rho_{11}+2 \rho_{12}+\rho_{22}\right) u_{x}^{2} .
$$

We conclude that

$$
\rho_{11}+2 \rho_{12}+\rho_{22}=\rho
$$

represents the total mass of the fluid-solid aggregate per unit volume. We may also express this quantity by means of the porosity $\beta$ and the mass densities $\rho_{8}$ and $\rho_{f}$ for the solid and fluid, respectively. The mass of solid per unit volume of aggregate is

$$
\rho_{1}=(1-\beta) \rho_{s},
$$

and the mass of fluid per unit volume of aggregate

Hence,

$$
\rho_{2}=\beta \rho_{f} \text {. }
$$

$$
\rho=\rho_{1}+\rho_{2}=\rho_{s}+\beta\left(\rho_{f}-\rho_{s}\right) \text {. }
$$

Let us now again assume that there is no relative motion between solid and fluid. The pressure difference in the fluid per unit length is

or

$$
-\frac{\partial p}{\partial x}=\rho_{f} \frac{\partial^{2} u_{x}}{\partial t^{2}}
$$

$$
-\frac{\partial p}{\partial x} \beta=\beta \rho_{f} \frac{\partial^{2} u_{x}}{\partial t^{2}} \text {. }
$$

The left-hand side is the force $Q_{x}$ acting on the fluid per unit volume. Hence, taking into account Eq. (3.7), we may write

$$
Q_{x}=\rho_{2} \frac{\partial^{2} u_{x}}{\partial t^{2}}
$$

Now, in the case

$$
u_{x}=U_{x},
$$

the second equation (3.3) becomes

$$
\left(\rho_{12}+\rho_{22}\right) u_{x}=Q_{x} \text {. }
$$

Comparing Eqs. (3.11) and (3.10) we derive

$$
\rho_{2}=\rho_{12}+\rho_{22} \text {. }
$$

Combining this result with relations (3.5) and (3.8) we have also

$$
\rho_{1}=\rho_{11}+\rho_{12} \text {. }
$$

The coefficient $\rho_{12}$ represents a mass coupling parameter between fluid and solid. This is illustrated by assuming that in some way the fluid is restrained so that the average displacement of the fluid is zero, i.e., $U_{x}=0$. Equations (3.3) are then written,

$$
\begin{array}{r}
q_{x}=\rho_{11} \frac{\partial^{2} u_{x}}{\partial t^{2}} \\
Q_{x}=\rho_{12} \frac{\partial^{2} u_{x}}{\partial t} .
\end{array}
$$

The second equation (3.14) shows that when the solid is accelerated a force $Q_{x}$ must be exerted on the fluid to prevent an average displacement of the latter. This effect is measured by the "coupling" coefficient $\rho_{12}$.The force $Q_{x}$ necessary to prevent the fluid displacement is obviously in a direction opposite to the acceleration of the solid; hence, we must always have

$$
\rho_{12}<0 \text {. }
$$

The same conclusion is reached by considering the first equation (3.10) in which $\rho_{11}$ represents the total effective mass of the solid moving in the fluid. This total mass must be equal to the mass proper of the solid $\rho_{1}$ plus an additional mass $\rho_{a}$ due to the fluid.

$$
\rho_{11}=\rho_{1}+\rho_{a} .
$$

From Eqs. (3.13) and (3.16) we derive

$$
\rho_{12}=-\rho_{a} \text {. }
$$


Hence, $\rho_{12}$ is the additional apparent mass with a change in sign. Therefore, the dynamic coefficients may be written

$$
\begin{aligned}
& \rho_{11}=\rho_{1}+\rho_{a} \\
& \rho_{22}=\rho_{2}+\rho_{a} \\
& \rho_{12}=-\rho_{a} .
\end{aligned}
$$

Further conditions must be satisfied by these dynamic coefficients if the kinetic energy is to be a positive definite quadratic form. The coefficients $\rho_{11}$ and $\rho_{22}$ must be positive

$$
\rho_{11}>0 \quad \rho_{22}>0
$$

and

$$
\rho_{11} \rho_{22}-\rho_{12}{ }^{2}>0 \text {. }
$$

These inequalities are always satisfied if the coefficients are given by the relations (3.18) where $\rho_{1} \rho_{2}$ and $\rho_{a}$ are positive by their physical nature.

In terms of stresses the force components are expressed as stress gradients, i.e.,

$$
\begin{aligned}
q_{x} & =\frac{\partial \sigma_{x}}{\partial x}+\frac{\partial \tau_{z}}{\partial y}+\frac{\partial \tau_{y}}{\partial z} \\
Q_{x} & =\partial s / \partial x, \text { etc. }
\end{aligned}
$$

Hence, we have the dynamic equations

$$
\begin{aligned}
\frac{\partial \sigma_{x}}{\partial x}+\frac{\partial \tau_{z}}{\partial y}+\frac{\partial \tau_{y}}{\partial z} & =\frac{\partial^{2}}{\partial t^{2}}\left(\rho_{11} u_{x}+\rho_{12} U_{x}\right) \\
\frac{\partial s}{\partial x} & =\frac{\partial^{2}}{\partial t^{2}}\left(\rho_{12} u_{x}+\rho_{22} U_{x}\right), \text { etc. }
\end{aligned}
$$

Strictly speaking, the generalized forces are defined as the virtual work of the microscopic stresses per unit value of the displacement vector $\mathbf{u}$ and $\mathbf{U}$ and not as the average of the microscopic stresses as used in expressions (3.20). However, for all practical purposes it is justified to use either definition.

It is interesting to note that because of the coupling coefficient an acceleration of the solid without average motion of the fluid produces a pressure gradient in the fluid. This is physically caused by an apparent mass effect of the fluid on the solid.

Equations (3.21) are referred to the $x$-direction. Identical equations may be written for the $y$ - and $z$-directions.

\section{EQUATIONS OF PROPAGATION OF PURELY ELASTIC WAVES}

Equations for the wave propagation are obtained by substituting expressions (2.12) for the stresses into the dynamical relations (3.21). We obtain for the $x$ - direction,

$$
\begin{array}{r}
N \nabla^{2} u_{x}+(A+N) \frac{\partial e}{\partial x}+Q \frac{\partial \epsilon}{\partial x}=\frac{\partial^{2}}{\partial t^{2}}\left(\rho_{11} u_{x}+\rho_{12} U_{x}\right) \\
Q \frac{\partial e}{\partial x}+R \frac{\partial \epsilon}{\partial x}=\frac{\partial^{2}}{\partial t^{2}}\left(\rho_{12} u_{x}+\rho_{22} U_{x}\right)
\end{array}
$$

and two other similar equations, respectively, for the directions $y$ and $z$. With the vector notation

$$
\begin{gathered}
\mathbf{u}=\left(u_{x} u_{y} u_{z}\right) \\
\mathbf{U}=\left(U_{x} U_{y} U_{z}\right) .
\end{gathered}
$$

Equations (5.1) are written

$$
\begin{aligned}
N \nabla^{2} \mathbf{u}+\operatorname{grad}[(A+N) e+Q \epsilon] & =\frac{\partial^{2}}{\partial t^{2}}\left(\rho_{11} \mathbf{u}+\rho_{12} \mathrm{U}\right) \\
\operatorname{grad}[Q e+R \epsilon] & =\frac{\partial^{2}}{\partial t^{2}}\left(\rho_{12} \mathbf{u}+\rho_{22} \mathbf{U}\right) .
\end{aligned}
$$

These six equations for the six unknown components of the displacements $\mathbf{u}$ and $\mathbf{U}$ completely determine the propagation.

Because of the statistical isotropy of the material, it can be shown that the rotational waves are uncoupled from the dilatational waves and obey independent equations of propagation. This is done in the usual way by introducing the operations div and curl

$$
\begin{array}{cc}
\operatorname{div} \mathbf{u}=\boldsymbol{e} & \operatorname{div} \mathrm{U}=\boldsymbol{\epsilon} \\
\operatorname{curlu}=\boldsymbol{\omega} & \operatorname{curl} \mathrm{U}=\mathbf{\Omega} .
\end{array}
$$

Applying the divergence operation to both equations (4.2), we obtain

$$
\begin{aligned}
& \nabla^{2}(P e+Q \epsilon)=\frac{\partial^{2}}{\partial t^{2}}\left(\rho_{11} e+\rho_{12} \epsilon\right) \\
& \nabla^{2}(Q e+R \epsilon)=\frac{\partial^{2}}{\partial t^{2}}\left(\rho_{12} e+\rho_{22} \epsilon\right)
\end{aligned}
$$

with the definition

$$
P=A+2 N \text {. }
$$

These two equations govern the propagation of dilatational waves. As discussed in more detail in the next section, it can already be seen that, in general, there will be two such dilatational waves and that each of these waves involves coupled motion in the fluid and the solid.

Similarly, applying the curl operation to equations (4.2) we obtain

$$
\begin{aligned}
& \frac{\partial^{2}}{\partial t^{2}}\left(\rho_{11} \omega+\rho_{12} \Omega\right)=N \nabla \omega \\
& \frac{\partial^{2}}{\partial t^{2}}\left(\rho_{12} \omega+\rho_{22} \Omega\right)=0 .
\end{aligned}
$$


These equations govern the propagation of pure rotational waves. It is seen that these equations imply also a coupling between the rotation $\omega$ of the solid and that of the fluid $\mathbf{\Omega}$. These equations will now be discussed.

\section{PROPERTIES OF THE PURELY ELASTIC WAVES}

Consider first, Eqs. (4.6) for the rotational waves. By eliminating $\boldsymbol{\Omega}$ in these equations, we find

$$
N \nabla^{2} \omega=\rho_{11}\left(1-\frac{\rho_{12}^{2}}{\rho_{11} \rho_{2}}\right) \frac{\partial^{2} \omega}{\partial t^{2}} .
$$

There is only one type of rotational wave. The velocity of propagation of these waves is

$$
V_{s}=\left[\frac{N}{\rho_{11}\left(1-\rho_{12}{ }^{2} / \rho_{11} \rho_{22}\right)}\right]^{\frac{1}{2}} .
$$

The rotation $\omega$ of the solid is coupled proportionally to the rotation $\boldsymbol{\Omega}$ of the fluid according to the relation

$$
\boldsymbol{\Omega}=-\frac{\rho_{12}}{\rho_{22}} \boldsymbol{\omega} .
$$

Since $\rho_{12}$ is negative and $\rho_{22}$ positive, the rotation of the fluid and the solid are in the same direction. This means that a rotation of the solid causes a partial rotational entrainment of the fluid through an inertia coupling. It this coupling did not exist and the fluid would stay at rest on the average the rotational wave velocity would be $V_{s}=\left(N / \rho_{11}\right)^{\frac{3}{3}}$, where $\rho_{11}$ represents the mass of the solid plus the apparent mass due to the relative motion of the solid in the fluid. Actually, the partial rotational entrainment of the fluid by the solid decreases the apparent mass effect with a corresponding increase in the wave velocity. This decrease of the apparent mass is expressed by the factor $\left[1-\left(\rho_{12}{ }^{2} / \rho_{11} \rho_{22}\right)\right]$ in formula (5.2).

The existence of a rotation in the fluid seems at first sight to be in contradiction with Kelvin's theorem that in a frictionless fluid without body forces no circulation can be generated. However, the velocity field $U$ here considered is not the actual microscopic velocity but the average volume flow. The circulation of the former remains zero in conformity with Kelvin's theorem while the line integral of the volume flow can be different from zero. The distinction is the same as made when we consider the apparent rotational inertia of a body immersed in a fluid. A rotation of the body in the fluid produces an angular momentum in the fluid while the circulation remains zero.

We now consider the dilatational waves defined by Eqs. (4.4). All essential features are brought out by discussing the propagation of a plane wave parallel with the $y z$-plane and of normal displacement $u_{x}$ and $U_{x}$ in the $x$-direction for the solid and the fluid, respectively.

It is convenient to introduce a reference velocity $V_{\text {。 }}$ defined by

$$
V_{c}^{2}=H / \rho,
$$

with $H=P+R+2 Q$. Referring to the definition of $\rho$ as representing the mass per unit volume of the fluidsolid aggregate it is seen by adding Eqs. (4.4) that $V_{o}$ represents the velocity of a dilatational wave in the aggregate under the condition that $e=\epsilon$, i.e., if the relative motion between fluid and solid were completely prevented in some way. The following nondimensional parameters are further introduced

$$
\begin{array}{ccc}
\sigma_{11}=\frac{P}{H} & \sigma_{22}=\frac{R}{H} & \sigma_{12}=\frac{Q}{H} \\
\gamma_{11}=\frac{\rho_{11}}{\rho} & \gamma_{22}=\frac{\rho_{22}}{\rho} & \gamma_{12}=\frac{\rho_{12}}{\rho} .
\end{array}
$$

The $\sigma_{i j}$-parameters define the elastic properties of the material while the $\gamma_{i j}$-parameters define its dynamic properties. Since we have the identities

$$
\sigma_{11}+\sigma_{22}+2 \sigma_{12}=\gamma_{11}+\gamma_{22}+2 \gamma_{12}=1 \text {, }
$$

there are only four independent parameters. We note that the positive character of the kinetic and elastic energies imply that $\sigma_{11} \sigma_{22}-\sigma_{12}{ }^{2}$ and $\gamma_{11} \gamma_{22}-\gamma_{12}{ }^{2}$ are positive.

With these parameters, Eqs. (4.4) are

$$
\begin{aligned}
& \nabla^{2}\left(\sigma_{11} e+\sigma_{12} \epsilon\right)=\frac{1}{V_{c}^{2}} \frac{\partial^{2}}{\partial t^{2}}\left(\gamma_{11} e+\gamma_{12} \epsilon\right) \\
& \nabla^{2}\left(\sigma_{12} e+\sigma_{22} \epsilon\right)=\frac{1}{V_{c}^{2}} \frac{\partial^{2}}{\partial t^{2}}\left(\gamma_{12} e+\gamma_{22} \epsilon\right) .
\end{aligned}
$$

Solutions of these equations are written in the form

$$
\begin{aligned}
& e=C_{1} \exp [i(l x+\alpha t)] \\
& \epsilon=C_{2} \exp [i(l x+\alpha t)] .
\end{aligned}
$$

The velocity $V$ of these waves is

$$
V=\alpha / l \text {. }
$$

This velocity is determined by substituting expressions (5.8) into (5.7).

Putting

we obtain

$$
z=V_{o}^{2} / V^{2}
$$

$$
\begin{aligned}
& z\left(\sigma_{11} C_{1}+\sigma_{12} C_{2}\right)=\gamma_{11} C_{1}+\gamma_{12} C_{2} \\
& z\left(\sigma_{12} C_{1}+\sigma_{22} C_{2}\right)=\gamma_{12} C_{1}+\gamma_{22} C_{2} .
\end{aligned}
$$

Eliminating $C_{1}$ and $C_{2}$ yields an equation for $z$,

$$
\begin{array}{r}
\left(\sigma_{11} \sigma_{22}-\sigma_{12}{ }^{2}\right) z^{2}-\left(\sigma_{11} \sigma_{22}+\sigma_{22} \gamma_{11}-2 \sigma_{12} \gamma_{12}\right) z \\
+\left(\gamma_{11} \gamma_{22}-\gamma_{12}\right)=0 .
\end{array}
$$


This equation has two roots $z_{1} z_{2}$ corresponding to two velocities of propagation $V_{1}, V_{2}$,

$$
\begin{aligned}
& V_{1}^{2}=V_{o}^{2} / z_{1} \\
& V_{2}^{2}=V_{c}^{2} / z_{2} .
\end{aligned}
$$

There are therefore two dilatational waves. The roots $z_{1}, z_{2}$ are always positive, since the matrices of coefficients $\sigma$ and $\gamma$ of Eqs. (5.11) are symmetric and are associated with positive definite quadratic forms representing respectively the potential and kinetic energies.

There are also orthogonality relations verified by the amplitude of these waves. Denote by $C_{1}{ }^{(1)} C_{2}{ }^{(1)}$ the amplitudes associated with the root $z_{1}$ and $C_{1}{ }^{(2)} C_{2}{ }^{(2)}$ the amplitudes associated with $z_{2}$. The orthogonality relations may be written

$$
\begin{aligned}
\gamma_{11} C_{1}{ }^{(1)} C_{1}{ }^{(2)}+2 \gamma_{12}\left(C_{1}{ }^{(1)} C_{2}{ }^{(2)}+C_{2}{ }^{(1)} C_{1}{ }^{(2)}\right)+ & \\
\gamma_{22} C_{2}{ }^{(1)} C_{2}{ }^{(2)} & =0 .
\end{aligned}
$$

This relation shows that if the amplitudes are of the same sign for one velocity say $V_{1}$ they are of opposite sign for the other. In other words, there is a wave in which the amplitudes are in phase and another in which they are in opposite phase. Moreover, from Eq. (5.11) we derive the relation

$$
z_{i}=\frac{\gamma_{11} C_{1}{ }^{(i) 2}+2 \gamma_{12} C_{1}{ }^{(i)} C_{2}{ }^{(i)}+\gamma_{22} C_{2}{ }^{(i) 2}}{\sigma_{11} C_{1}{ }^{(i) 2}+2 \sigma_{12} C_{1}{ }^{(i)} C_{2}{ }^{(i)}+\sigma_{22} C_{2}{ }^{(i) 2}} .
$$

Because $\gamma_{12}$ is the only negative coefficient, we conclude that the higher velocity has amplitudes in phase while the low velocity has amplitudes in opposite phase.

It is interesting to note the possible existence of a wave such that $\mathbf{u}=\mathbf{U}$, i.e., for which there is no relative motion between fluid and solid. By putting $C_{1}=C_{2}$ in Eq. (5.12) it is seen that this occurs if the parameters of the material satisfy the relation

$$
\frac{\sigma_{11}+\sigma_{12}}{\gamma_{11}+\gamma_{12}}=\frac{\sigma_{22}+\sigma_{12}}{\gamma_{22}+\gamma_{12}}=1 \text {. }
$$

This may be called a "dynamic compatibility" relation. It will be shown below that it plays an important role in the case where dissipation is considered. The propagation velocity for this case is given by

$$
V_{1}^{2}=H / \rho \text {. }
$$

There is another wave propagation with a lower velocity $V_{2}$. It may be verified from the orthogonality relation (5.14) that if Eq. (5.16) is satisfied the amplitude ratio for this wave is,

$$
C_{1}{ }^{(2)} / C_{2}{ }^{(2)}=-\rho_{2} / \rho_{1} \text {. }
$$

It is also easily shown that the compatibility relation (5.16) is a necessary and sufficient condition for one root of Eq. (5.12) to be equal to unity.

As a matter of terminology the high-velocity dilata- sional wave will be designated as the wave of the first kind and the low-velocity wave as the wave of the second kind. ${ }^{2}$

\section{EQUATIONS OF PROPAGATION WHEN DISSIPATION IS INTRODUCED}

It will be assumed that the flow of the fluid relative to the solid through the pores is of the Poiseuille type. That this assumption is not always valid is well known, e.g., when the Reynolds number of the relative flow exceeds a certain critical value. The assumption also breaks down when we exceed a certain characteristic frequency. If we accept the assumption of Poiseuille flow the microscopic flow pattern inside the pores is uniquely determined by the six generalized velocities

$$
\dot{u}_{x}, \dot{u}_{y}, \dot{u}_{z}, \dot{U}_{x}, \dot{U}_{y}, \dot{U}_{z} .
$$

As before, the kinetic energy depends only on these six coordinates. Dissipation depends only on the relative motion between the fluid and the solid. Introducing the concept of dissipation function, we may write this function as a homogeneous quadratic form with the foregoing six generalized velocities. Because of the assumed statistical isotropy, orthogonal directions are uncoupled. The dissipation also vanishes when there is no relative motion of fluid and solid; hence, when

$$
\dot{u}_{x}=\dot{U}_{x} \quad \dot{u}_{y}=\dot{U}_{y} \quad \dot{u}_{z}=\dot{U}_{z} .
$$

The dissipation function $D$ is therefore

$$
2 D=b\left[\left(\dot{u}_{x}-\dot{U}_{x}\right)^{2}+\left(\dot{u}_{y}-\dot{U}_{y}\right)^{2}+\left(\dot{u}_{z}-\dot{U}_{z}\right)^{2}\right] .
$$

Lagrange's equation with a dissipation function are written (for the $x$-direction)

$$
\begin{gathered}
\frac{\partial}{\partial t}\left(\frac{\partial T}{\partial \dot{u}_{x}}\right)+\frac{\partial D}{\partial \dot{u}_{x}}=q_{x} \\
\frac{\partial}{\partial t}\left(\frac{\partial T}{\partial \dot{U}_{x}}\right)+\frac{\partial D}{\partial \dot{U}_{x}}=Q_{x} .
\end{gathered}
$$

Explicitly, we have

$$
\begin{aligned}
& \frac{\partial^{2}}{\partial t^{2}}\left(\rho_{11} u_{x}+\rho_{12} U_{x}\right)+b \frac{\partial}{\partial t}\left(u_{x}-U_{x}\right)=q_{x} \\
& \frac{\partial^{2}}{\partial t^{2}}\left(\rho_{12} u_{x}+\rho_{22} U_{x}\right)-b \frac{\partial}{\partial t}\left(u_{x}-U_{x}\right)=Q_{x} .
\end{aligned}
$$

We have seen that the generalized forces are related to the stresses by relations (3.20). The dynamic equations in terms of the stress components are

$$
\begin{aligned}
\frac{\partial \sigma_{x}}{\partial x}+\frac{\partial \tau_{z}}{\partial y}+\frac{\partial \tau_{y}}{\partial z} & =\frac{\partial^{2}}{\partial t^{2}}\left(\rho_{11} u_{x}+\rho_{12} U_{x}\right)+b \frac{\partial}{\partial t}\left(u_{x}-U_{x}\right) \\
\frac{\partial s}{\partial x} & =\frac{\partial^{2}}{\partial t^{2}}\left(\rho_{12} u_{x}+\rho_{22} U_{x}\right)-b \frac{\partial}{\partial t}\left(u_{x}-U_{x}\right) .
\end{aligned}
$$


Expressing the stresses in terms of the displacements $\mathbf{u}$ and $\mathbf{U}$ the relations (6.6) become

$$
\begin{aligned}
N \nabla^{2} \mathbf{u}+\operatorname{grad}[(A+N) e+Q \epsilon] & \\
& =\frac{\partial^{2}}{\partial t^{2}}\left(\rho_{11} \mathbf{u}+\rho_{12} \mathbf{U}\right)+b \frac{\partial}{\partial t}(\mathbf{u}-\mathbf{U}) \\
\operatorname{grad} & {[Q e+R \epsilon] } \\
& =\frac{\partial^{2}}{\partial t^{2}}\left(\rho_{12} \mathbf{u}+\rho_{22} \mathbf{U}\right)-b \frac{\partial}{\partial t}(\mathbf{u}-\mathbf{U}) .
\end{aligned}
$$

The coefficient $b$ is related to Darcy's coefficient of permeability $k$ by

$$
b=\mu \beta^{2} / k,
$$

where $\mu$ is the fluid viscosity and $\beta$ the porosity as defined in Sec. 2.

We have mentioned in the foregoing that the assumption of Poiseuille flow breaks down if the frequency exceeds a certain value. This is illustrated by considering a plane boundary in the presence of an infinitely extended vicous fluid and oscillating harmonically in its own plane. The velocity parallel with the plane at a distance $y$ from the plane is

$$
u=\exp \left[i \alpha t-(1+i)\left(\frac{\alpha}{2 \nu}\right)^{\frac{1}{2}} y\right]
$$

where $\nu=\mu / \rho_{f}$ is the kinematic viscosity. The quarter wavelength of the boundary layer is

$$
y_{1}=\pi(\nu / 2 \alpha)^{\frac{1}{2}} .
$$

For a porous material we may assume that Poiseuille flow breaks down when this quarter wavelength is of the order of the diameter $d$ of the pores, i.e., for frequencies higher than

$$
f_{t}=\pi \nu / 4 d^{2} .
$$

In the case of water at $15^{\circ} \mathrm{C}$ we find $f_{t}=100 \mathrm{cps}$ for $d=10^{-2} \mathrm{~cm}$ and $f_{t}=10^{4} \mathrm{cps}$ for $d=10^{-3} \mathrm{~cm}$.

\section{NUMERICAL DISCUSSION OF ATTENUATED WAVES}

As in the case of purely elastic waves, we may separate the body waves into uncoupled rotational and dilatational waves. Applying the divergence operator to Eqs. (6.7) we find the equations for dilatational waves

$$
\begin{aligned}
& \nabla^{2}(P e+Q \epsilon)=\frac{\partial^{2}}{\partial t^{2}}\left(\rho_{11} e+\rho_{12} \epsilon\right)+b-\frac{\partial}{\partial t}(e-\epsilon) \\
& \nabla^{2}(Q e+R \epsilon)=\frac{\partial^{2}}{\partial t^{2}}\left(\rho_{12} e+\rho_{22} \epsilon\right)-b \frac{\partial}{\partial t}(e-\epsilon) .
\end{aligned}
$$

Similarly, applying the curl operator, we have the equation for rotational waves

$$
\begin{aligned}
& \frac{\partial^{2}}{\partial t^{2}}\left(\rho_{11} \omega+\rho_{12} \Omega\right)+b \frac{\partial}{\partial t}(\omega-\Omega)=N \nabla^{2} \omega \\
& \frac{\partial^{2}}{\partial t^{2}}\left(\rho_{12} \omega+\rho_{22} \Omega\right)-b-\frac{\partial}{\partial t}(\omega-\Omega)=0 .
\end{aligned}
$$

Consider first a rotational plane wave propagating in the $x$-direction. The magnitude of the rotations of solid and fluid in the $z$-direction may be put equal to

$$
\begin{aligned}
& \omega=C_{1} \exp [i(l x+\alpha t)] \\
& \Omega=C_{2} \exp [i(l x+\alpha t)] .
\end{aligned}
$$

It is convenient to introduce a characteristic frequency

$$
f_{c}=\frac{b}{2 \pi \rho_{2}}=\frac{b}{2 \pi \rho\left(\gamma_{12}+\gamma_{22}\right)} .
$$

Introducing the solution (7.3) into the propagation solutions (7.2) and eliminating the constants $C_{1}, C_{2}$ we find

with

$$
N l^{2} / \rho \alpha^{2}=E_{r}-i E_{i}
$$

$$
\begin{gathered}
E_{r}=\frac{1+\gamma_{22} \frac{\gamma_{11} \gamma_{22}-\gamma_{12}{ }^{2}}{\left(\gamma_{12}+\gamma_{22}\right)^{2}}\left(\frac{f}{f_{c}}\right)^{2}}{1+\left(\frac{\gamma_{22}}{\gamma_{12}+\gamma_{22}}\right)^{2}\left(\frac{f}{f_{c}}\right)^{2}} \\
E_{i}=\frac{f}{f_{c}} \frac{\left(\gamma_{12}+\gamma_{22}\right)}{1+\left(\frac{\gamma_{22}}{\gamma_{12}+\gamma_{22}}\right)^{2}\left(\frac{f}{f_{c}}\right)^{2}}
\end{gathered}
$$

the frequency of the wave is $f=\alpha / 2 \pi$. Putting

$$
l=l_{r}+i l_{i}
$$

the phase velocity is

$$
v_{r}=\alpha /\left|l_{2}\right| \text {. }
$$

We introduce a reference velocity

$$
V_{r}=(N / \rho)^{\frac{1}{2}}
$$

which is the velocity of rotational waves for the case of no relative motion between fluid and solid. We derive from (7.5)

$$
\frac{v_{r}}{V_{r}}=\sqrt{2} /\left[\left(E_{r}^{2}+E_{i}^{2}\right)^{\frac{3}{2}}+E_{r}\right]^{\frac{1}{3}} .
$$

This velocity ratio is a function only of the frequency ratio $f / f_{c}$ and the dynamic parameters $\gamma_{i j}$. The velocity ratio is plotted in Fig. 1 for cases 1 to 4 of Table $I$.

It will be noted that there are only two independent 


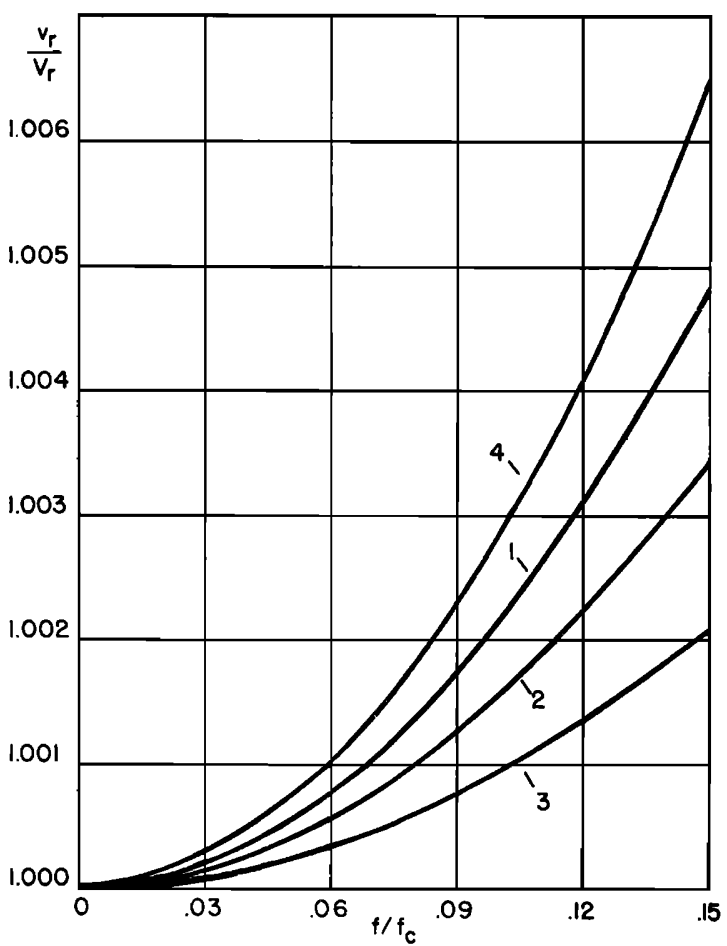

FIG. 1. Phase velocity $\nu_{r}$ of rotational waves.

parameters since they must satisfy relation (5.7). According to the previous discussion of Sec. 6, the Poiseuille flow assumption is valid below a certain frequency. If we introduce the assumption that the pores behave like circular tubes of diameter $d$ we may put

$$
b=32 \mu \beta / d^{2} \text {. }
$$

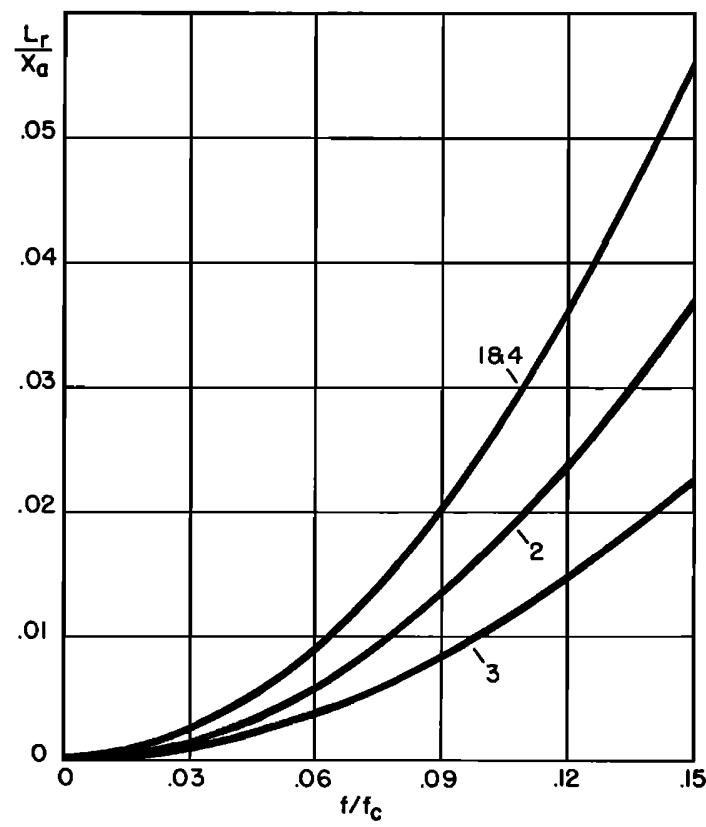

FIG. 2. Attenuation coefficient of rotational waves.
In this case

$$
\frac{f_{t}}{f_{c}}=\frac{\pi^{2}}{64}=0.154 .
$$

Therefore, the curves are plotted only in the range

$$
0<f / f_{0}<0.15 \text {. }
$$

The attenuation of the rotational waves is determined by $l_{i}$. With a reference length

and putting

$$
L_{r}=\frac{V_{r}}{2 \pi f_{c}}
$$

we find

$$
x_{a}=1 /\left|l_{i}\right| \text {, }
$$

$$
\frac{x_{a}}{L_{r}}=\sqrt{2} \frac{f_{c}}{f}\left[\left(E_{r}^{2}+E_{i}^{2}\right)^{\frac{1}{2}}-E_{r}\right]^{-\frac{1}{1}} .
$$

The amplitude of the wave as a function of the distance $x$ is proportional to $\exp \left(-x / x_{a}\right)$. The nondimensional attenuation coefficient $L_{r} / x_{a}$ is plotted in Fig. 2 for cases 1 to 4 of Table $I$.

TABLE I.

\begin{tabular}{ccccccccc}
\hline \hline Case & $\sigma_{11}$ & $\sigma_{22}$ & $\sigma_{12}$ & $\gamma_{11}$ & $\gamma_{22}$ & $\gamma_{12}$ & $z_{1}$ & $z_{2}$ \\
\hline 1 & 0.610 & 0.305 & 0.043 & 0.500 & 0.500 & 0 & 0.812 & 1.674 \\
2 & 0.610 & 0.305 & 0.043 & 0.666 & 0.333 & 0 & 0.984 & 1.203 \\
3 & 0.610 & 0.305 & 0.043 & 0.800 & 0.200 & 0 & 0.650 & 1.339 \\
4 & 0.610 & 0.305 & 0.043 & 0.650 & 0.650 & -0.150 & 0.909 & 2.394 \\
5 & 0.500 & 0.500 & 0 & 0.500 & 0.500 & 0 & 1.000 & 1.000 \\
6 & 0.740 & 0.185 & 0.037 & 0.500 & 0.500 & 0 & 0.672 & 2.736 \\
\hline
\end{tabular}

Approximate formulas may be obtained by expanding expressions (7.10) and (7.15) for small values of $f / f_{c}$.

We find

$$
\begin{aligned}
& \frac{v_{r}}{V_{r}}=1+\frac{1}{8}\left[4 \gamma_{22}-\left(\gamma_{12}+\gamma_{22}\right)^{2}\right]\left(\frac{f}{f_{c}}\right)^{2} \\
& \frac{L_{r}}{x_{a}}=\frac{1}{2}\left(\gamma_{12}+\gamma_{22}\right)\left(\frac{f}{f_{c}}\right)^{2} .
\end{aligned}
$$

When plotted these formulas give values indistinguishable from the exact graph for the range $f / f_{c}<0.15$.

We now consider dilatational waves. They are governed by Eqs. (7.1). Again we consider plane waves and put

$$
\begin{aligned}
& \boldsymbol{e}=C_{1} \exp [i(l x+\alpha t)] \\
& \boldsymbol{\epsilon}=C_{2} \exp [i(l x+\alpha t)] .
\end{aligned}
$$

Substitution in Eqs. (7.1) and elimination of the constants $C_{1}, C_{2}$ yield the relation

$$
\begin{gathered}
\left(P R-Q^{2}\right) \frac{l^{4}}{\alpha^{4}}-\left(R \rho_{11}+P \rho_{22}-2 Q \rho_{12}\right) \frac{l^{2}}{\alpha^{2}}+\rho_{11} \rho_{22}-\rho_{12}{ }^{2} \\
+\frac{i b}{\alpha}\left[(P+R+2 Q) \frac{l^{2}}{\alpha^{2}}-\rho\right]=0 .
\end{gathered}
$$


With the variables already introduced in Sec. (5) this equation may be written in nondimensional form

$\left(\sigma_{11} \sigma_{22}-\sigma_{12}^{2}\right) z^{2}-\left(\sigma_{22} \gamma_{11}+\sigma_{11} \gamma_{22}-2 \sigma_{12} \gamma_{12}\right) z$

with

$$
+\left(\gamma_{11} \gamma_{22}-\gamma_{22}\right)^{2}+\frac{i b}{\alpha \rho}(z-1)=0
$$

$$
z=\frac{l^{2}}{\alpha^{2}} V_{c}^{2}
$$

In this case $l$ and hence $z$ are complex. If we put $b=0$ in Eqs. (7.20) we obtain Eq. (5.12) whose roots

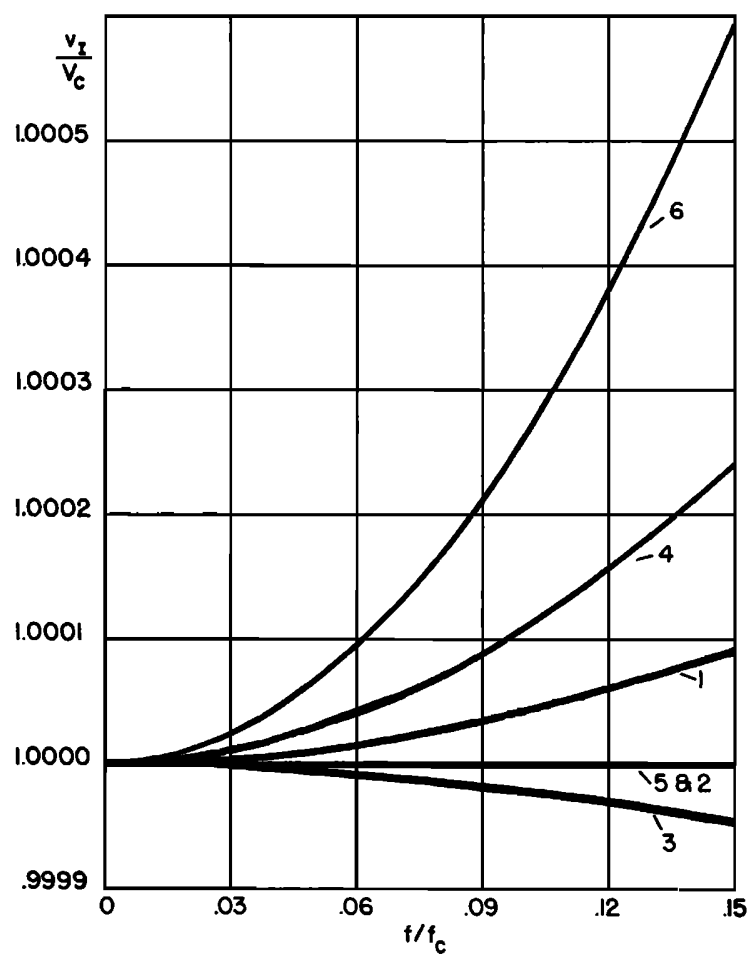

Fig. 3. Phase velocity $v_{l}$ of dilatational waves of the first kind.

are $z_{1}, z_{2}$. With these roots Eq. (7.20) may be written,

where

$$
\left(z-z_{1}\right)\left(z-z_{2}\right)+i M(z-1)=0
$$

$$
M=\frac{b}{\alpha \rho\left(\sigma_{11} \sigma_{22}-\sigma_{12}{ }^{2}\right)} .
$$

The roots $z$ of Eq. (7.22) yield the properties of the dilatational waves as a function of a frequency variable $M$ and two parameters $z_{1}, z_{2}$ which correspond to the velocities of the purely elastic waves as given by Eq. (5.13). We may write $M$ in terms of $f / f_{c}$ as

$$
M=\frac{f_{c}}{f} \frac{\left(\gamma_{12}+\gamma_{22}\right)}{\left(\sigma_{11} \sigma_{22}-\sigma_{12}{ }^{2}\right)} .
$$

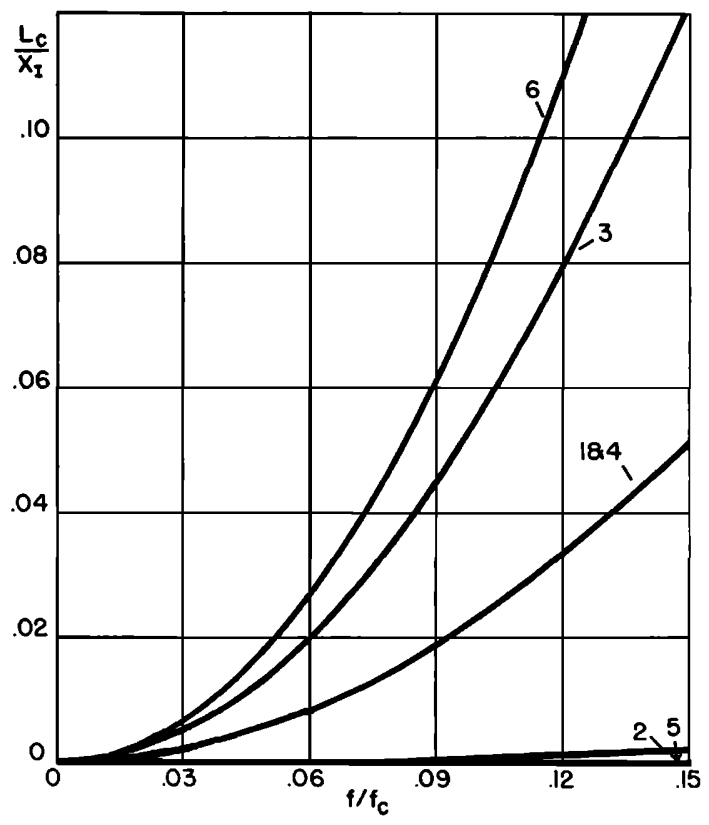

Fig. 4. Attenuation coefficient of dilatational waves of the first kind.

We denote by $z_{I}$ and $z_{I I}$ the roots of Eq. (7.22). We assume that $z_{I}$ is the root which tends to unity at zero frequency $(f=0)$. Then this root corresponds to waves of the first kind while $z_{I I}$ corresponds to waves of the second kind. We also write

$$
\begin{aligned}
\left(z_{I}\right)^{\frac{1}{2}} & =R_{I}+i \mathcal{T}_{I} \\
\left(z_{I I}\right)^{\frac{1}{3}} & =\mathcal{R}_{I I}+i \mathcal{T}_{I I} .
\end{aligned}
$$

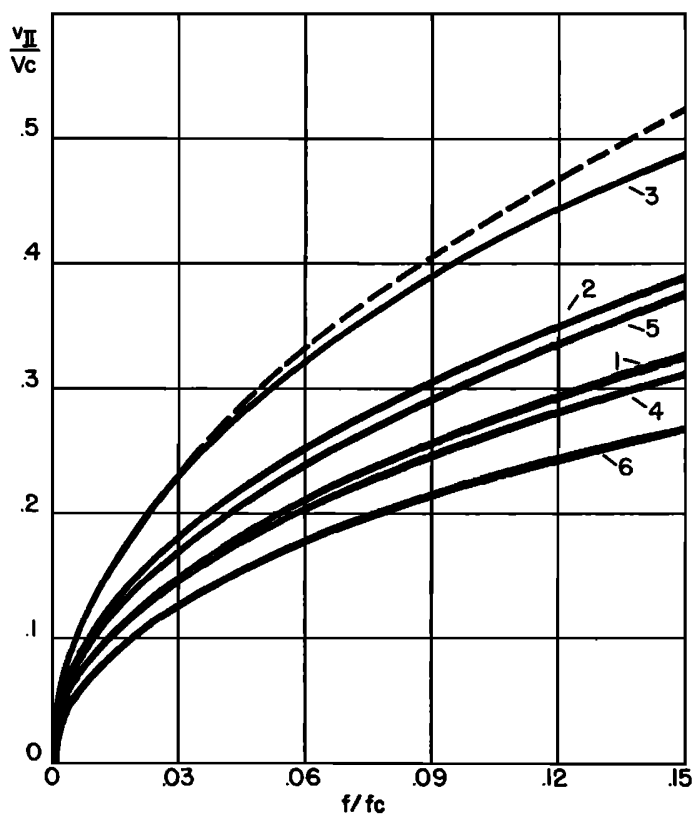

FIG. 5. Phase velocity $v_{I I}$ of dilatational waves of the second kind. 


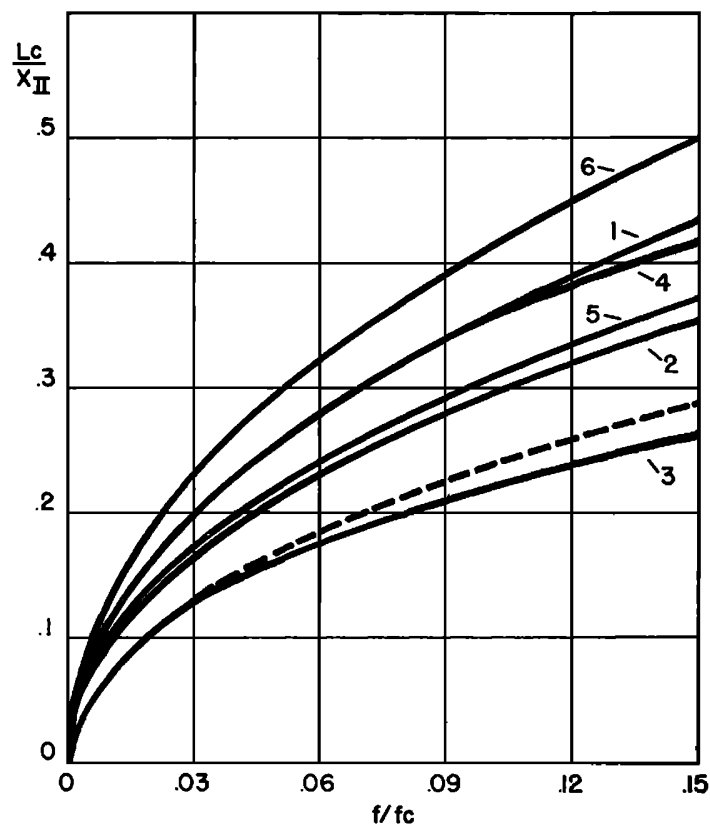

Fig. 6. Attenuation coefficient of dilatational waves of the second kind.

The phase velocity $v_{I}$ of the waves of the first kind is given by

$$
v_{I} / V_{c}=1 /\left|\mathbb{R}_{I}\right| \text {. }
$$

The ratio $v_{I} / V_{c}$ is plotted as a function of the frequency ratio $f / f_{c}$ in Fig. 3 for the six cases of Table I. The numbers in all figures refer to the six cases listed in Table I.

Attenuation of the waves of the first kind is given by

$$
L_{c} / x_{I}=\left|T_{I}\right|\left(f / f_{c}\right)
$$

and plotted in Fig. 4. The amplitude of the wave as a function of the distance is proportional to $\exp \left(-x / x_{I}\right)$ and $L_{c}$ is a characteristic distance given by

$$
L_{c}=\frac{V_{c}}{2 \pi f_{c}} .
$$

The phase velocity and attenuation of the wave of the second kind are similarly given by

$$
\begin{aligned}
& \frac{v_{I I}}{V_{c}}=\frac{1}{\left|R_{I I}\right|} \\
& \frac{L_{c}}{x_{I I}}=\left|\mathcal{T}_{I I}\right| \frac{f}{f_{c}} .
\end{aligned}
$$

These are plotted in Figs. 5 and 6.

Expanding expressions (7.26) and (7.27) for small values of the frequency variable $f / f_{c}$, then putting

$$
\begin{aligned}
& \zeta_{1}=z_{1}-1 \\
& \zeta_{2}=z_{2}-1,
\end{aligned}
$$

we find the following approximations for the waves of the first kind

$$
\begin{aligned}
& \frac{v_{I}}{V_{c}}=1-\frac{1}{2}\left(\frac{f}{f_{c}}\right)^{2} \frac{\left(\sigma_{11} \sigma_{22}-\sigma_{12}\right)^{2}}{\left(\gamma_{12}+\gamma_{22}\right)^{2}} \zeta_{1} \zeta_{2}\left(\zeta_{1}+\zeta_{2}+\frac{1}{2} \zeta_{1} \zeta_{2}\right) . \\
& \frac{L_{c}}{x_{I}}=\frac{1}{2}\left|\zeta_{1} \zeta_{2}\right| \frac{\sigma_{11} \sigma_{22}-\sigma_{12}{ }^{2}}{\gamma_{12}+\gamma_{22}}\left(\frac{f}{f_{c}}\right)^{2} .
\end{aligned}
$$

These approximates are quite satisfactory when compared with the exact values.

Similarly, for the waves of the second kind we have the approximations

$$
\begin{aligned}
& \frac{v_{I I}}{V_{c}}=\left(2 \frac{f}{f_{c}} \frac{\left(\sigma_{11} \sigma_{22}-\sigma_{12}{ }^{2}\right)}{\left(\gamma_{12}+\gamma_{22}\right)}\right)^{\frac{t}{2}} \\
& \frac{L_{c}}{x_{I I}}=\left(\frac{1}{2} \frac{f}{f_{c}} \frac{\left(\gamma_{12}+\gamma_{22}\right)}{\left(\sigma_{11} \sigma_{22}-\sigma_{12}{ }^{2}\right)}\right)^{\frac{1}{3}} .
\end{aligned}
$$

The approximations (7.34), (7.35) are plotted as dotted lines for case 3 in Figs. 5 and 6 . The departure from the exact value is small. This has also been verified for the other cases. These approximations correspond to diffusion type propagation with an attenuation factor per cycle equal to exp $(-2 \pi)$. In the lowfrequency range therefore the waves of the second kind behave like a diffusion or heat conduction phenomenon and the attenuation is very high. 